ஜாஹிலிய்யாக் கால அரேபிய இலக்கியத்தில் உலகப்பற்றின்மை

வெளிப்பாடுகள்: குஸ் பின் ஸாஇதாவின் கருத்துக்களை முதன்மைப்படுத்திய ஆய்வு

PM. Hamthoon a, *

a Faculty of Arts\& Culture, Eastern University, Sri Lanka.

*Corresponding author Email: hamthoonpms@esn.ac.lk

DOI: https://doi.org/10.54392/ijmrd2143

Received: 01-09-2021; Revised: 25-11-2021; Accepted: 26-11-2021 Published: 29-11-2021

Abstract: Jahillayath means ignorance. The Arabic word Jahiliyyah refers to the zealous culture and civilized society in the Islamic case. It is against Islam. The Jahiliyya community is a brutal society with human characteristics cut off. Gus bin Zaydah was a literary figure who lived in the so-called Jahiliyya social period. It can be observed that Islamic thought is often exaggerated in his poetry and prose literature. Much of his literature, prose and poetry, speaks of the triviality of worldly life and the permanence of the afterlife. Death is expressed in many of his speeches and poems. This is in stark contrast to pagan literature. Therefore, this study seeks to introduce Jahiliyyah and express the uniqueness of Arabic literature and to reveal the secular expressions of thought in the literary aspects of the Jahiliyya period writer Gus bin Zaydah. For this purpose descriptive and analytical methods were used and studied.

Keywords: Secularism, Arabic Literature, Jahiliyyah Period, Gus Bin Zaydah

Language: Tamil

முன்னுரை

இலக்கியம் ஒரு சமூகத்தின் அடையாளம். அது அச்சமூகத்தின் வரலாற்று மூலமும் கூட. அது குறித்த சமூகத்தைப் பிரதிபலிக்கின்றது. இலக்கியத்தினூடாக வளர்ச்சி பெறாத எந்தவொரு சமூகமும் காலப்போக்கில் அழிந்தே போயுள்ளன. சமூகத்தினது இருப்பும் இயங்கியலும் இலக்கியத்திலேயே தங்கியுள்ளது. இதனால்தான் உலகில் தோன்றிய சமூகங்கள் தமது இலக்கியத்தைத் தனித்துவமாகக் கட்டியெழுப்ப முயற்சித்துள்ளன.

“இலக்கியத்தின் இலக்கணத்தை மிகத் துல்லியமாக வரையறுத்து விளக்கம் தருவது கடினமாகும். அழகு, கவிதை, கற்பனை, குறிக்கோளியல் முதலியவற்றை எவ்வாறு துல்லியமாக விளக்க இயலாதோ அவ்வாறே இலக்கியத்தையும் விளக்க இயலாது என்பார் வின்செஸ்டர். ஆயினும் அறிஞர்கள் கூறும் வரையறைகளிலிருந்து அதன் பொருளை விளங்கிக் கொள்ளலாம். இலக்கியமானது சிறந்த கருத்துகள் அடங்கிய நூலாகும் என்பார் எமர்சன். படிப்போர்க்கு இன்பம் தரும் வகையில் நுண்ணறிவு வாய்ந்த அறிஞர்கள் தமது உணர்ச்சிகளையும் கருத்துக்களையும் முறைப்படுத்தி எழுதுவது இலக்கிமாகும் என்கிறார் ஸ்போர்டு புரூக். தன்கண் அமைந்த நுவல் பொருளும் அதை விளக்கும் முறையும் பொதுவாக மக்களைக் கவரும் வகையில் அமைந்து, வடிவம் இன்றியமையாததாகி அதன் வாயிலாக இன்பம் நல்குவது இலக்கியமாகும் என்று டபிள்யூ.எச் அட்சன் குறிப்பிடுகிறார்." (Kathiresan, 2016)

Indian J Multiling Res Dev., 2(4) (2021), 19-24 | 19 
இலக்கியங்கள் பாரிய சமூகப் பணிகளை ஆற்றுகின்றன. அது சமூகத்தின் குறுக்குவெட்டு முகமாகத் தொழிற்படுகின்றது. இலக்கியம் என்பது சமுதாய நிலைமைகளினின்றும் ஊற்றெடுப்பது அந்த வகையிலே அதுவும் வாழ்க்கையிலே ஓர் அம்சமாக அதன் ஓர் அங்கமாக இருக்கிறது. வாழ்க்கையை இயக்கும் மிகப் பிரதானமான சக்தியாக அது இல்லையாயினும் அதுவும் கணிசமான தாக்கத்தை உடைய ஒரு துணைவலுவாக உள்ளது. இலக்கியங்கள் வெளிப்படையாகவும் நேரடியாகவும் அபிப்பிராயங்களை மாற்றுவதில்லை. ஆயினும், அவை சிறுகச் சிறுக, மெல்ல மெல்ல நம்மைத் தங்கள் பக்கம் இழுத்து வசப்படுத்திக் கொள்ளும் ஆற்றல் உடையவை. ஆகவேதான் விழுமிய இலட்சியங்களுடன் ஒன்றி நின்று அந்த இலட்சிய மணங்கமழும் ஓர் உலகினை இடையறாது கண்டு காட்டும் ஓர் இலட்சியப் படைப்பாளி தன் வாசகர்களின் மனப்பழக்கத்திலும் கணிசமானதொரு மாற்றத்தை உண்டு பண்ணுவான். இலக்கியப் படைப்பாளி உண்டு பண்ணும் இம்மாற்றம் மானசிகமானது மனத்தளவில் நின்று விடுவது. மானசிகமான இந்த அபிப்பிராய மாற்றம் தன்னளவிலே அதிகம் பயன்படாது. மானசிக மாற்றங்களுக்கு ஈடாகப் புறவுலகிலும் மாற்றங்கள் உண்டாக வேண்டும். மானசிக எழுச்சி புறவுலக நடைமுறை உலகினால் மெய்ப்பிக்கப்படுகையில், மேலும் மேலும் மானசிக மாற்றங்களும் புற உலக மாற்றங்களும் நேரும். சமுதாயப் பிரச்சினைகளுக்கு ஏற்ற தீர்வுகள் மேற்படி மாற்றங்களின் திரட்சியினாலும் வளர்ச்சியினாலுமே கிட்டும்." (Murukaiyan, 1988)

இத்தகைய கருத்துருவாக்கப் பணியை அரபு இலக்கியத்திலும் காணலாம். அரபு இலக்கியம் பிளவுபட்ட சமூககக் கடட்மைப்பிலிருந்து ஐக்கியத்தின் பால் கொண்டு சென்றது. அரேபிய இலக்கியத்தைப் படிக்கும் ஒருவர் மன நிறைவைப் பெறும் நிலை தோன்றுகின்றது. அது மனித இலயல்புடன் ஓன்றித்துச் செல்கின்றது. அது இலக்கியத்திற்கும் சமூகத்துக்கிடையிலான முரண்பாட்டைத் தவிர்த்து இணைப்பை ஏற்படுத்துகின்றது. "அரபு இலக்கியம் பண்டைய அரேபிய மொழியினதும் அதன் சமூகத்தினதும் இலக்கியமாகும். அரேபிய தீபகற்பத்தில் சமூகங்களை ஒன்று சேர்த்த இஸ்லாத்தின் வருகைக்குப் பின்னரே அதன் நிஜ வடிவம் தோற்றம் பெற்றது. இதன் மூலம் பல கோத்திரங்களாக இருந்த அவர்கள் பூரண சமூகமாக மாறினர். இது பண்டைய அரேபிய கவிதை மற்றும் அதனுடன் தொடர்புடைய குறிசொல்லல் இலக்கிய தொகுப்புக்கள் இருந்தது என்ற கருத்தை தடுக்காது." இக்கருத்து அரேபிய இலக்கியம் இஸ்லாத்திற்கு முன்னரும் பின்னரும் காலத்திற்கு ஏற்ப சமூக இலக்கியமாக வளர்ச்சி பெற்றது என்பதை சுட்டி நிற்கின்றது (Aljundiu, 1985).

\section{ஆய்வு பிரச்சினை}

ஜாஹிலியா என்றால் அறியாமை மடமை என்று பொருளாகும். ஜாஹிலிய்யத் என்ற அரபுப் பதம் இஸ்லாமிய வழக்கில் சீரிய பண்பாடு, நாகரிகம் அறிவு இல்லாத சமூகத்தை குறித்து நிற்கின்றது. மனித பண்புகள் அற்றுப்போய் மிருகத்தனமான சமூகமே ஜாஹிலிய்ய சமூகம் ஆகும். இவ்வாறு அறியப்பட்ட ஜாஹிலிய்ய சமூகக் காலத்தில் வாழ்ந்த ஓர் இலக்கியவாதி தான் குஸ் பின் ஸாஇதா. இவருடைய கவிதை மற்றும் உரைநடை இலக்கியங்களில் பெரும்பாலும் இஸ்லாமிய சிந்தனை மிகைத்து காணப்படுவதை அவதானிக்கலாம். இவருடைய இலக்கியங்களான உரைநடை மற்றும் கவிதைகளில் அதிகமானவை உலக வாழ்வின் அற்பத்தன்மை, மறுமை வாழ்வின் நிரந்தரத் தன்மை குறித்து பேசுகின்றன. இவருடைய அதிகமான உரைகளிலும் கவிதைகளிலும் மரணம் குறித்து வெளிப்படுத்தப்படுகின்றது. இது ஜாஹிலிய்ய இலக்கியத்தில் இருந்து மாறுபட்ட தன்மை கொண்டதாக உள்ளது. எனவே இவரது சிந்தனைக்கும் இஸ்லாமிய சமய சிந்தனைக்கும் இடையிலான தொடர்பினை கண்டறிந்தது அதன் பின்னணியிலுள்ள காரணம் கண்டறியப்படல் வேண்டும்.

\section{ஆய்வு நோக்கம்}

1. ஜாஹிலிய்யத்தை அறிமுகம் செய்தல்.

2. அரேபிய இலக்கியத்தின் தனித்துவத்தை வெளிப்படுத்தல் 
3. ஜாஹிலிய்யா கால இலக்கியவாதியான குஸ் பின் ஸாஇதாவை அறிமுகம் செய்து அவரது இலக்கிய அம்சங்களிலுள்ள உலகப்பற்றற்ற சிந்தனை வெளிப்பாடுகளை வெளிக்கொணர்தல்.

\section{ஆய்வு முறையியல்}

ஆய்வு நோக்கங்களை அடைந்து கொள்வதற்காக விவரண மற்றும் பகுப்பாய்வியல் முறைகள் பயன்படுத்தப்பட்டன. முதலாம் நிலைத்தரவுகளுடன் இரண்டாம் நிலைத்தரவுகளாக நூல்கள், சஞ்சிகைள், பத்திரிகைகள் மற்றும் இணையம் போன்றவற்றின் மூலம் தரவுகள் பெறப்பட்டு ஆய்வுக்குட்படுத்தப்பட்டன.

\section{பெறுபேறுகளும் கலந்துரையாடலும்}

1) ஜாஹிலிய்யா அரேபிய இலக்கியம்

இஸ்லாத்தின் வருகைக்கு முந்திய இலக்கிய படைப்புக்கள் ஜாஹிலிய்ய இலக்கியம் எனப்படுகின்றது. “கி.பி. 5ஆம் நூற்றாண்டின் நடுப்பகுதியில் ெென் தேசத்தவர்களிடமிருந்து அங்கு வசித்த அத்னான் கோத்திரத்தவர்கள் சுதந்திரம் பெற்றுச் சென்றதிலிருந்து ஜாஹிலிய்யாக் காலம் ஆரம்பித்து கி.பி. 622இல் இஸ்லாம் தோன்றியதுடன் முடிகின்றது." என அஹ்மத் ஹஸன் அஸ்ஸெய்யாத் தன்னுடைய தாரீகுல் அதபில் அரபி என்ற நூலில் (பக்: 6) குறிப்பிடுகின்றார்.

“அரேபியாவில் இஸ்லாத்திற்கு முந்திய (இந்த ஜாஹிலிய்யா) இலக்கியம் பெரும்பாலும் கவிதையைத்தனித்துவமாகக் கொண்டது. நாடகம், காவியம் என்பன கவிதையைத்தனித்துவமாகக் கொண்டது. நாடகம், காவியம் என்பன அவர்களிடத்தில் இருக்கவில்லை. அவர்களின் கவிதைகள் பாடல்கவிதை (Lyrical Poetry) வடிவில் அமைந்தவை. இப்பாடல்கவிதைகள் காவியங்களுக்குரிய நாடகங்களுக்கரிய சில கூறுகளைப் பிரதிபலித்தன என்பர். தொன்மைக்காலத்தில் நாடங்களில் வளர்ச்சிக்கும் புராணவியலுக்கும் நெருங்கிய சம்பந்தமிருந்துள்ளன. அரேபிய மரபில் சிக்கல்மிகுந்த புராணவியல் இருக்கவில்லை. அரேபியரின், கடின வாழ்க்கை முறையும் பாலைவனச் சுழலும் நாடகம், காவியம் போன்ற கலைகளின் வளர்ச்சிக்கு சாதகமானதாக அமையவில்லை. அரேபியரின் மனப்பாங்கு மிகக்கூரிய தனிநபர் தன்மை கொண்டது. இதனால் தனிநபர் மனோநிலைகளைக் கூறக்கூடிய சிறு பாடல் வகைகளில் அவர்கள் அதிக நாட்டம் செலுத்தினர். இவ்வகையிலான சிந்துகளும் கஸீதாக்களும் அவனுக்குக் கைவந்த கலைகளாகின. இவை செபிப்புல வாய்ப்பாட்டுக் கவிதைகள், இவற்றுக்கு எழுத்து வடிவம் இல்லை. பரம்பரை பரம்பரையாக இக்கவிதைகள் வாய்மாறிச் சென்றன. இப்பாடல் கவிதைகளுக்குக் குரல் உயிர் மூச்சாகும். இவை பேச்சாகவோ அல்லது பேச்சுநிலை கடந்ததாகவோ ஆனால் குரலுக்கும் மொழிக்குமிடையிலான செழுமையையும் சிக்கலும் நிறைந்த தொடர்பின் பிறப்பிடமாகவோ இருந்தன என்பர் எடொனிஸ். அரபு மக்களின் வழக்காறுகள், மரபுகள், வீரப்பிரதாபங்கள் துயரங்கள், தோல்விகள் முதலியவற்றை இக்கவிதைகள் வெளிப்படுத்தின. வாய்வழியாகக் கவிதையைக் கேட்பதையே முந்தைய அரேபியர் தகுந்த வடிவமெனக்கருதினர். அரபு மொழியில் பாடல் என்பது குரலையே வேர்ச் சொல்லாகக் கொண்டிருந்தது. வாய்வழியாய்க் கவிதை இசைத்தலை தனித்துவனமான கலையென அரேபியர் கருதினர். வருடாந்த சந்தைகள் கூடும்போது வர்த்தக நடவடிக்கையோடு கவிதை வெளியீடுகளும் வேறு பொழுதுபோக்குகளும் இடம்பெற்றன. மக்காவுக்கு அருகே இருந்த 'உக்காஸ்’ இத்தகைய நிகழ்ச்சிகளுக்கு பெயர்பெற்று விளங்கியது. கவிஞர்கள் கவிதைகளை இங்கு வெளியிட்டனர். பேச்சாளர் தமது மொழி அலங்கார வல்லமையைக்காட்டினர். வருவதுரைப்போரும். குறிசொல்வோரும் நடக்க இருப்பன பற்றி எடுத்துரைத்தனர்." (Anas, 1997) 
2) இலக்கியத்தில் உலகப்பற்றின்மை

“உலகப்பற்றின்மை என்பது அல்லாஹ்வை விட்டு தூரப்படுத்தும் பாவங்களிலிருந்து தூரமாகி, உலக விவகாரங்களிலிருந்து முழுமையாக ஒதுங்கி இருப்பதாகும். அது உள்ளத்தைக் பரிசுத்தப்படுத்துவதை மூலம் ஏற்படும். ேேுதல் மற்றும் போதுமென்ற மனநிலை என்பவற்றை விடவும் ஆழமான கருத்தைக் கொண்டது. உலகப்பற்றின்மை என்ற கருத்துப் போக்கு ஒன்றுக்கொன்று பலமான தொடர்பினைக் கொண்டுள்ள ஆன்மீன, லௌகீக, பண்பியல் கருத்துக்களை உள்ளடக்குகின்றது. அடியான் அல்லாஹ் செய்யக் கூடாதென்று தடுத்த காரியத்தில் ஈடுபட்ட நிலையில் உலகப்பற்றில்லாதவனாக முடியாது. எனவே, ஆன்மாவும் சிந்தனையும் ஒன்றிணையாமல் உலகப்பற்றின்மை கிடையாது.' (Farhan, 2013)

உலகப்பற்றற்ற நிலையை ஆர்வமூட்டும் பல கருத்துக்களை அரபு இஸ்லாமிய இலக்கியங்கள் கொண்டுள்ளன. "இஸ்லாம் துறவறத்தை அங்கீகரிக்கவில்லை. அதேவேளை உலக வாழ்வில் தீவிர ஈடுபாடு கொண்டு அதிலே மூழ்கி விடுவதையும் அது ஏற்பதில்லை. இவ்விரு போக்குகளுக்கும் இடைப்பட்ட சமநிலையான போக்கைக் கையாளுமாறும் அது போதிக்கிறது. உலக கவர்ச்சிகளுக்கு முன்னால் நிலைகுலைந்து அவற்றின் பின்னால் ஓடுபவனை உலகாயதவாதி எனலாம். அதேநேரம் உலக வாழ்வை முற்றாகத் துறந்து செல்பவனை துறவி எனலாம். இவ்விரு தீவிர நிலைகளுக்குமிடையே ஒரு சமநிலையான போக்கைக் கடைப்பிடிப்பவனே ஸாஹித் அல்லது ஸஹ்த் உள்ளவனென இஸ்லாம் கூறுகிறது. உலகவாழ்வு அற்பமானது அதனது இன்பங்கள் அற்பமானது என்ற கருத்தை அல்குர்ஆன் பல இடங்களில் விளக்கியுள்ளது. அவ்வசனங்களைச் சரிவரப் புரிந்துகொள்வதனூடாக ஸஹ்த் பற்றிய இஸ்லாத்தின் கண்ணோட்டத்தைப் புரிந்து கொள்ளலாம். 'உலக வாழ்க்கை வீண் விளையாட்டும் கேளிக்கைகளுமாகும். தக்வா உள்ளவர்களுக்கு மறுமையே

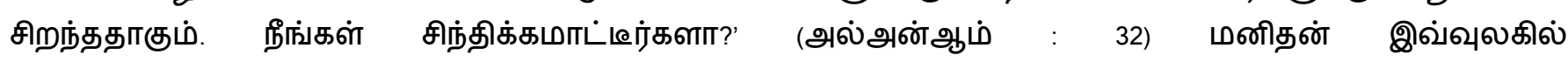
சோதனைக்காகவே படைக்கப்பட்டிருக்கிறான். எனவே இவ்வுலக வாழ்வை ஒரு பரீட்சைக்களமாகக் கருதி நடந்துகொள்ள வேண்டும் என்ற கருத்தை வலியுறுத்துவதனூடாகவும் அவனிடத்தில் பற்றற்ற தன்மையை வளர்த்து விட இஸ்லாம் முயற்சி செய்கிறது. ‘உங்களில் எவர் செயலில் மிகச் சிறந்தவர் எனச் சோதிப்பதற்காக அவன் வாழ்வையையும், மரணத்தையும் படைத்தான்.' அல் முல்க் : 2" (NIE, 2017).

\section{3) குஸ் பின் ஸாஇதாவும் உலகப்பற்றற்ற சிந்தனையும்}

குஸ் பின் ஸாஇதா அரேபிய ஜாஹிலிய்யா சமூகத்தில் வாழ்ந்த பிரபல்யமான இலக்கியவாதி. இவர் உரைநடை இலக்கியத்திற்கு பெயர்போனவர். கவிதைகளும் பாடியுள்ளார். இவரது இலக்கியத்தில் பெரும்பாலும் சமயம்சார் அல்லது உலகப்பற்றற்ற வாழ்வியல் தொடர்பான சிந்தனைகiளே காணப்படும். “இவர் நஜ்ரான் பகுதியைச் சேர்ந்த முன்னைய இறை வேதங்களைக்கற்ற மதப்போதகராகவும் அரேபிய சொற்பொழிவாளராகவும் ஞானியாகவும் மத்தியஸ்தம் செய்பவராகவும் இருந்தார். அல்லாஹ்வை விசுவாசம் கொண்டு அவன் பக்கம் ஞானத்துடனும் நல்லுபதேசம் செய்தார். உயரத்தில் நின்று வாளை ஊன்றியவராக உரையாற்றியவரும் உரையில் 'அம்மா பஃது' என்ற வார்த்பையைப் பயன்படுத்திய முதலாமவராகக் கருதப்படுகின்றார். இவர் மன்னர் கைஸரை அடிக்கடி சந்திப்பார். மன்னரும் இவரை மிகுந்த கண்ணியப்படுத்துவார். ஆனாலும் உலகப்பற்றற்றவராகவே வாழ்ந்துள்ளார். கி.பி. 600இல் மரணித்தார்." (As'seyyāt, Tārīkul atapil arapi).

ஒரு முறை குஸ் பின் ஸாஇதாவின் கோத்திரத்தவர்கள் நபி (ஸல்) அவர்கனைச் சந்திக்க வந்தனர். அப்போது நபியவர்கள் குஸ் பின் ஸாஇதா ஏதாவது ‘வஸிய்யத்' (மரண சாசனம்) செய்திருந்தாரா எனக் கேட்டார்கள். அவரது தலைப் பக்கத்தில் ஒரு ஏட்டைக் கண்டதாகவும் அதில் அவர் பின்வரும் கவிதையை எழுதியிருந்ததாகவும் சொன்னார்கள் 


$$
\begin{aligned}
& \text { يا ناعي الموت و الأموات في جدث عليهم من بقايا نومهم خرق } \\
& \text { دعهم فإن لهم يوما يصاح بهم كما ينبه من نوماته الصعق } \\
& \text { منهم عراة وموتى في ثيابهر منها الجديد ومنها الأزرق الخلق }
\end{aligned}
$$

மரணச் செய்தியை கொண்டு வருபவனே! மரணித்தவர்கள் மண்ணறையிலே எஞ்சிய அவர்கள தூக்கத்தினால் எதுவுமே செய்ய முடியாதோராக உள்ளனர். அவர்களை விட்டு விடு. அவர்கள் எழுப்பப்படும் ஒரு நாளுண்டு. பலத்த சத்தம் அவர்களை எழுப்பும். அவ்வாறு எழுப்பப்படுவோரில் ஆடையிலுள்ளோரும் ஆடையற்றவர்களும் இருப்பர். அவ்வாடைகளில் புதியதும் நீலநிற உருவங்களும் இருக்கும்." (Imām ipñu kasīr, 1990)

குஸ் பின் ஸாஇதாவின் அதிக கவிதைகளில் மரணம் குறித்தும் மனித வாழ்வின் நிலையற்ற தன்மை குறித்தும் அதிகளவில் பேசப்பட்டுள்ளது. மற்றொரு எடுத்துக்காட்டு:

$$
\begin{aligned}
& \text { في الذاهبين الأولي ن من القرون لنا بصائر }
\end{aligned}
$$

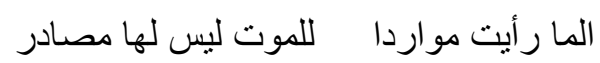

$$
\begin{aligned}
& \text { ورأيت قومي نحو ها يسعى الأصاغر و الأكابر }
\end{aligned}
$$

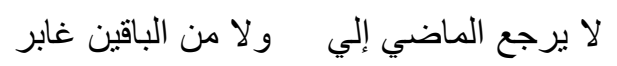

$$
\begin{aligned}
& \text { أيقنت أني لا محالة حيث صدار القوم صائر }
\end{aligned}
$$

“எனக்கு முன் மறைந்து சென்றவர்களில் நமக்கு படிப்பினைகள் உள்ளன. மரணத்தைக் கொண்டுவரும் மூலங்கள் எதையும் நான் பார்த்தில்லை. எனது சமூகத்திலுள்ள சிறியவர்களும் பெரியவர்களும் அதைநோக்கி விரைந்து செல்கின்றனர். சென்ற காலம் மீண்டும் எனக்கு வராது. எஞ்சியிருப்பவர்களில் தப்பிப்போர் யாரும் இல்லை. சமூகத்தில் நடப்பது நடக்கம் என்பதில் எந்தவொரு சந்தேகமுமில்லை என நான் உறுதியாக நம்புகின்றேன்." (Imām ipnu kasīr, 1990)

ஜாஹிலிய்யா கால சமூகத்தில் சந்தைகள் சிறப்புற்று விளங்கின. அதில் ‘உக்காஸ்’ சந்தை பிரபல்யமானது. அதில் குஸ் பின் ஸாஇதா உரையாற்றுபவராக இருந்தார். ஒரு முறை அவர் ஆற்றிய பின்வரும் உரையை வாலிபராக இருந்த நபி (ஸல்) அவர்களும் செவியுற்றார்கள்:

$$
\begin{aligned}
& \text { يا أيها الناس اجتمعو ا واستمعو ا وعوا ، من عاش مات ، و ، ومن مات فات ، و وكل ما هو آت آت ، إن في السماء لخبرا ، و وإن في الأرض }
\end{aligned}
$$

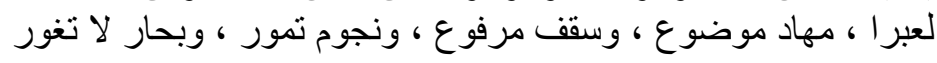

"மனிதர்களே வாருங்கள், நான் சொல்வதை கேட்டு ஞாபகம் வைத்துக்கொள்ளுங்கள். எவன் வாழ்ந்தானோ அவன் மரணித்தான். எவன் மரணித்தானோ அவன் தப்பி விட்டான். வந்தது வந்ததுதான். வானிலே செய்தியும் பூமியில் படிப்பினையும் உள்ளது. பூமி தாழ்த்தப்பட்டுள்ளது. வானம் உயர்த்தப்பட்டுள்ளது. நட்சத்திரங்கள் அசைகின்றன. கடல் ஓடுகின்றது." (Imām ipñu kasīr, 1990).

\section{முடிவுரை}

அரபிலக்கியம் ஒரு சமூக இலக்கியமாக சமூகத்தின் கருத்துக்களை வெளிப்படுத்துகின்ற ஒரு தளமாக இயங்கியுள்ளது. அரபு இலக்கியம் ஜாஹிலியா கால இலக்கியம், இஸ்லாமிய ஆரம்பகால இலக்கியம், அப்பாஸியர் கால இலக்கியம், துருக்கியர் கால இலக்கியம், நவீன இலக்கியம் என்று ஐந்து பகுதிகளாக பிரிக்கப்படுகின்றது. ஜாஹிலிய்யாக் கால இலக்கியம் பெரும்பாலும் ஜாஹிலிய்ய கால மக்களின் உணர்ச்சிகளை வெளிப்படுத்த கூடியவையாக அமைந்துள்ளன. ஜாஹிலியா காலம் என்றாலே பண்பாட்டு, ஒழுக்க வீழ்ச்சிமிக்க வாழ்வு முறை கொண்ட, சமய அறிவு இல்லாத ஒரு சமூகத்தை குறித்து நிற்கின்ற பதமாகும். இத்தகைய ஒரு சமூகத்திலிருந்து சமூகத்தை பிரதிநிதித்துவப்படுத்த வராக இருக்கிறார் குஸ் பின் ஸாஇதா. இவர் கி.பி. 600இல் மரணித்தார். இஸ்லாத்தை அறிமுகம் செய்த நபிகள் நாயகம் (ஸல்) அவர்கள் உயிரோடு இருந்த காலப்பகுதியில் இவர் வாழ்ந்திருக்கின்றார். 
முன்னைய வேதங்களைக் கற்று அல்லாஹ், மறுமையை நம்பி இருந்த ஒரு இறை விசுவாசியாக வாழ்ந்தார். ஜாலியா சமூகத்தை பிரதிநிதித்துவப் படுத்தினாலும் இஸ்லாமிய கருத்துக்களைக் கொண்ட ஒருவராக இருந்தார். இவருடைய உரைநடை மற்றும் கவிதை இலக்கியங்களில் இது வெளிப்படுவதை காணலாம். குறிப்பாக இவருடைய இலக்கிய அம்சங்களில் உலகப் பற்றற்ற தன்மை குறித்து அதிகம் பேசப்பட்டுள்ளது. மரணம், மரணத்துக்கு பின்னரான வாழ்வு, இவ்வுலகின் நிரந்தரமற்ற தன்மை குறித்து அதிகமாக பேசியுள்ளார். இத்தகைய மிகச் சிறந்த இலக்கியப் படைப்பாளிகளின் இலக்கிய அம்சங்களை பல்கலைக்கழக கல்விப் புலத்தில் அறிமுகம் செய்து வைப்பதன் ஊடாக அரேபிய இலக்கியத்தின் தனித்துவத்தையும் செழுமையையும் அடுத்த தலைமுறைக்கு கொண்டு செல்ல வேண்டுமென்று இவ்வாய்வு பிரேரிக்கின்றது.

\section{References}

Aljundiu, A., (1985). Khasayis Al'adab Aalearabii, Dar Alkitab Allubnaniu, Bayrut.

Anas, MSM., (1997). Islāttin tōrram oru camūka paṇpāțtịal āyvu, Cultural Research Circle, Peradeniya, Srilanka

As'seyyāt, A.H, (n.d). Tārīkul atapil arapi, Tārunnahl̄ā misr, Cairo

Farhan, B.H., (2013). Shaer alzuhdi- alnash'at waltatawur - dirasat tahliliat naqdia, Al- Adab Journal, 103, 66-92. https://www.iasj.net/iasj?func=fulltext\&aId $=83604$

Imām ipnnu kasīr, (1990). Alpitāyā vannihāyā, Vol 2, Maktapattu ma'ārip, Beirut

Kathiresan, K., (2016). Ilakkiyat tirannāyvu, Manonmaniam Sundaranar University, Tirunalveli, India

Murukaiyan, I., (1988). Iñraiya ulakil ilakkiyam, Chennai Books, Chennai, India

NIE, (2017). Islam G.C.E. A/L. TIM, National Intitute of Education, Maharagama, Srilanka.

\section{Funding}

No funding was received for conducting this study.

\section{Conflict of interest}

The Author has no conflicts of interest to declare that they are relevant to the content of this article.

\section{About the License}

(C) The author 2021. The text of this article is open access and licensed under a Creative Commons Attribution 4.0 International License

\section{Cite this Article}

PM. Hamthoon, The manifestations of secularism in the Arabic literature of the Jahiliyyah period: A study prioritizing the views of Gus Bin Zaydah, Vol 2, Iss 4 (2021) 19-24. DOI: https://doi.org/10.54392/ijmrd2143 